\title{
Loss of vascular early response gene reduces edema formation after experimental stroke
}

\author{
Fudong Liu', L Christine Turtzo ${ }^{1}$, Jun Li ${ }^{1}$, Jean Regard ${ }^{2}$, Paul Worley ${ }^{2,3}$, Neer Zeevi ${ }^{1,4}$ and Louise D McCullough ${ }^{1,4^{*}}$
}

\begin{abstract}
Vascular Early Response Gene (Verge) is an immediate early gene (IEG) that is up-regulated in endothelial cells in response to a number of stressors, including ischemic stroke. Endothelial cell lines that stably express Verge show enhanced permeability. Increased Verge expression has also been associated with blood brain barrier breakdown. In this study we investigated the role of Verge in ischemic injury induced by middle cerebral artery occlusion (MCAO) in both Verge knockout (KO) and wild type (WT) mice. Verge KO mice had significantly less cerebral edema formation after MCAO compared to WT mice. However, stroke outcome (infarct size and neurological deficit scores) evaluated at either 24 or 72 hours after stroke showed no differences between the two genotypes. Verge deletion leads to decreased edema formation after ischemia; however acute stroke outcomes were unchanged.
\end{abstract}

Keywords: Edema, Ischemic Stroke, Middle Cerebral Artery Occlusion (MCAO), Neurological Deficit Score

\section{Introduction}

The endothelial cells of the brain blood barrier (BBB) are the first line of the defense between the systemic circulation and the brain [1,2]. After stroke, changes in cell shape lead to a loosening of endothelial cell-cell contacts, impairing endothelial barrier function and increasing paracellular permeability. The subsequent development of tissue edema contributes to worsening of injury in many illnesses, including stroke [3]. Immediate early genes (IEG) are activated within minutes of stimulation and thus do not require de novo protein synthesis. They are critical in determining how gene transcription is controlled in response to extracellular signaling [4]. One of the initial genes of this class discovered was fos, which was found to be rapidly induced by growth factor treatment without the need for new protein synthesis [5]. IEGs such as c-jun and c-fos are involved in ischemic injury [6,7], and are strongly associated with downstream endothelial responses [8]. Verge is a novel IEG selectively induced in endothelial cells after ischemia or acute systemic hypertonicity, which has been associated with enhanced vascular permeability $[9,10]$. However, whether Verge contributes to the

\footnotetext{
* Correspondence: Imccullough@uchc.edu

'Department of Neuroscience, University of Connecticut Health Center, Farmington, CT 06030, USA

${ }^{4}$ Department Neurology, University of Connecticut Health Center, 263

Farmington Ave, Farmington, CT 06030, USA

Full list of author information is available at the end of the article
}

development of ischemic injury, or enhances strokeinduced $\mathrm{BBB}$ dysfunction is not clear. In this study, we used both Verge KO and C57BL6 WT littermate mice to test the hypothesis that deletion of Verge would reduce cerebral edema and improve stroke outcomes induced by MCAO.

\section{Materials and methods}

Both Verge KO (Original breeding pairs from John Hopkins University) and WT C57BL6 littermates (Charles River, Frederick, Maryland) were 10-12 weeks of age (20-25 g) when subjected to MCAO. Genotyping was performed by $\mathrm{PCR}$ with the following primers: $F 2$ 5' -CTCTAGCCTAGGGCAGCAAC-3'; wtR1 5' -GAGAGAGGTCGGACGTGATG-3'; LacZR 5'-GGCGATTAAGTTGGGTAACG-3' (Regard and Worley, unpublished data). This study was conducted in accordance with $\mathrm{NIH}$ guidelines for the care and use of animals in research and under protocols approved by the Animal Care and Use Committee of the University of Connecticut Health Center.

Cerebral ischemia was induced by 90 min of MCAO under isoflurane anesthesia, followed by reperfusion as described in [11] with a total survival time of $30 \mathrm{~min}$ utes, 24 or 72 hours. Protein assessment was performed by immunohistochemistry (IHC) and Western blots. In the IHC cohort, mice were sacrificed 30 minutes after 
stroke; then brains were perfused and stained with CD31 (eBioscience, San Diego, CA) and Verge antibodies as in $[9,12]$. In the Western cohort, protein was isolated from total brain 24 hours after stroke as previously described [13]. Brain homogenates were separated on $10-20 \%$ polyacrylamide gels (Bio-Rad, Hercules, CA), then blotted to PVDF membranes and probed using anti-Verge antibodies [9].

Brain edema was measured 24 hours after stroke as described previously [14]. Briefly, the brain was quickly removed after the animal was sacrificed. Then the brain was blotted to remove residual absorbent moisture, and dissected through the interhemispheric fissure into right and left hemispheres ( $\mathrm{n}=6$ animals/group). Wet weight was determined with a resolution of $0.1 \mathrm{mg}$. Dry weight of whole ipsilateral and contralateral hemispheres was determined after heating the tissue for 3 days at $100{ }^{\circ} \mathrm{C}$ in a drying oven. Absolute water content was calculated as $\% \mathrm{H}_{2} \mathrm{O}=(1-$ dry weight/wet weight $) \times 100 \%$. Relative water content was determined by the ratio of ipsilateral/ contralateral absolute water content.

To evaluate stroke outcomes, mice were euthanized 24 or 72 hours ( $\mathrm{n}=7 \sim 9$ animals/group) after MCAO for infarct volume analysis with 2,3,5-triphenyltetrazolium chloride (TTC) staining as in [11]. The infarct volumes (\% contralateral hemisphere structure, corrected for edema) were analyzed using computer software (Sigmascan Pro5) as previously described [14]. Neurological deficits (NDS) were scored 1.5 and 72 hours after initiation of MCAO with the scoring system described in [15]: 0 , no deficit; 1 , forelimb weakness and torso turning to the ipsilateral side when held by tail; 2 , circling to affected side; 3 , unable to bear weight on affected side; and 4, no spontaneous locomotor activity or barrel rolling. Investigators were blinded to mouse genotype for stroke surgery, behavioral testing and infarct analysis. All mice were screened prior to stroke for turning bias and were randomly assigned into each cohort. Data from individual experiments were presented as Mean \pm SEM and analyzed with a $t$-test. NDS were presented as Median (interquartile range) and analyzed with Mann-Whitney $U$ test. $P<0.05$ was considered statistical significant.

\section{Results}

We first confirmed gene expression of Verge in our model. PCR and Western Blotting results showed that Verge KO mice lacked the DNA for the WT Verge allele (Figure 1A) and did not express Verge protein (Figure 1B). A significant increase in Verge expression was seen in the brain after 24 hours of stroke compared to sham mice (Figure $1 \mathrm{~B} \& \mathrm{C}$ ). Immunofluorescent staining with antiVerge antibody showed selective staining of the endothelium, but not all vessels (identified by CD31+ staining) co-expressed Verge 30 minutes in the penumbra after stroke
(Figure 1D). Verge was not expressed in the brains of sham WT mice (data not shown).

Absolute water content in the ipsilateral (stroke) hemisphere of both $\mathrm{KO}$ and WT mice was significantly increased compared with that of the contralateral hemisphere after stroke (Figure 2A). Verge KO mice had significantly less water content in the ipsilateral hemisphere compared to WT mice. The absolute water content in the contralateral hemisphere of $\mathrm{KO}$ mice was also less than that in WT mice although this difference was not significant. We also calculated the relative water content, comparing each genotype to its contralateral (nonstroke) hemisphere, which showed no difference between KO and WT mice (Figure 2B).

Histological analysis revealed that infarct volumes in Verge $\mathrm{KO}$ mice were not significantly different from that of WT mice 24 hours after MCAO ( $>0.05$ ) (Figure 2C). As peak edema may occur later after injury, we also examined a large cohort of mice 72 hours after stroke. Neither were there difference in infarct size between $\mathrm{KO}$ and WT mice at 72 hours after stroke (Figure 2D). There were also no differences in NDS between KO and WT mice at $1.5 \mathrm{~h}$ or $72 \mathrm{~h}$ after MCAO, although both genotypes had significantly improved NDS at $72 \mathrm{~h}$ compared to deficits seen $1.5 \mathrm{~h}$ after stroke (Figure 2E).

\section{Discussion}

Verge is a novel IEG that can be rapidly induced in the mature cerebral vasculature by a variety of stimuli [9] To date, only one study [9] has examined the expression of Verge in the cerebral vasculature after ischemic injury, which reported both Verge mRNA and protein expression were increased in the brain 2 hours after a 90 minute ischemic insult. The up-regulation of Verge was confirmed in this study, in which we found increased Verge expression as early as 30 minutes after 90 minute focal stroke. Verge increases monolayer permeability in endothelial cells $[9,10]$ in vitro, however it is not yet known if Verge expression also leads to exacerbated edema formation in the brain after injury. Our study revealed that Verge $\mathrm{KO}$ mice had significantly less absolute water content in the ipsilateral hemisphere compared to WT mice, consistent with the concept that Verge expression leads to enhanced endothelial permeability. Interestingly, the relative water content showed no differences between $\mathrm{KO}$ and WT mice (Figure 2B). This suggests that stroke induces equivalent water accumulation in the ischemic brains of Verge $\mathrm{KO}$ and WT mice, and that the reduction in water content after stroke in $\mathrm{KO}$ mice was related more to loss of Verge rather than to a specific response to ischemic injury. Previous studies have shown that Verge functions to dynamically regulate the cellular osmoadaptation to 


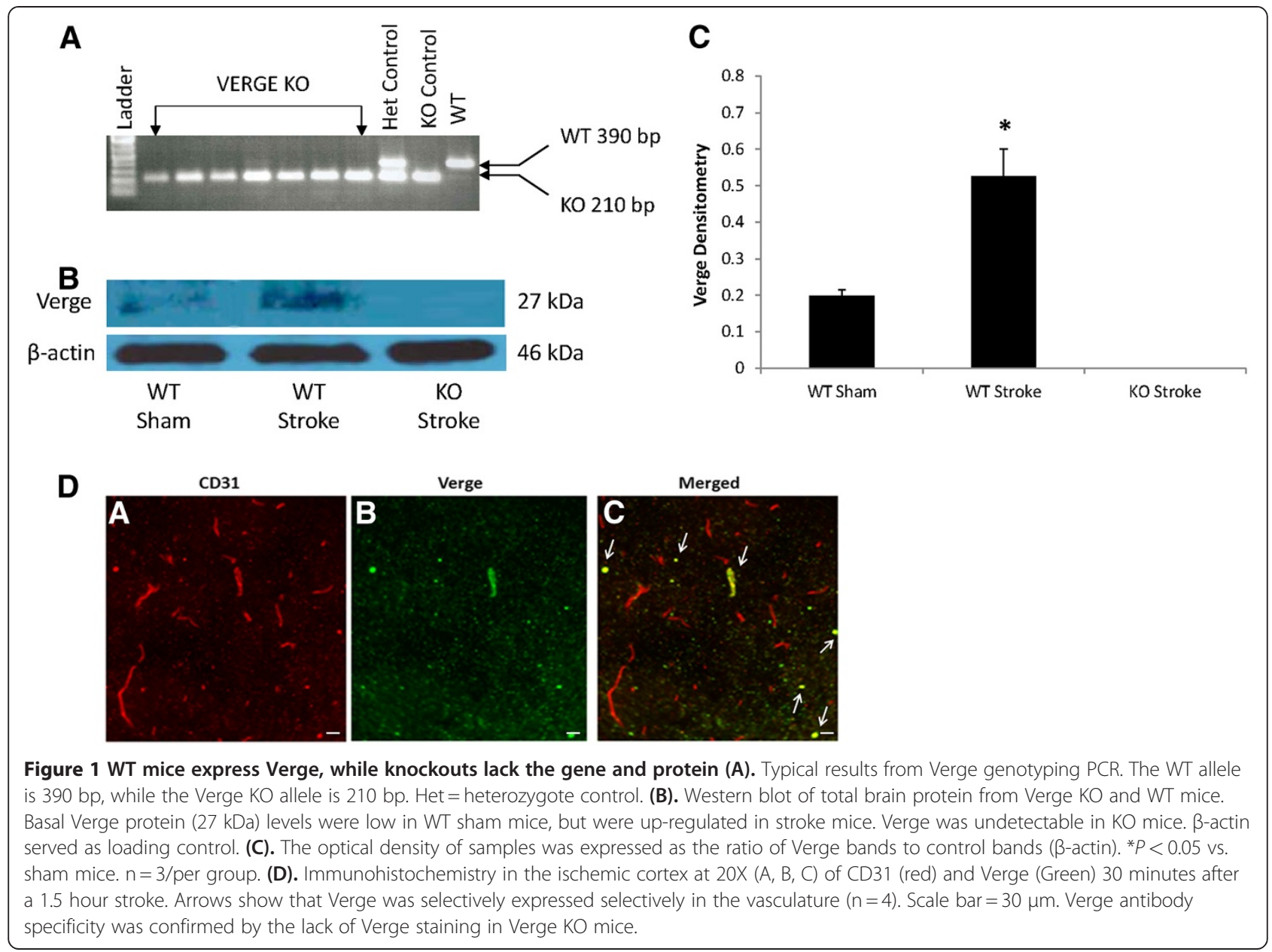

hypertonicity [10]. This effect is mediated by protein kinase C-dependent signaling which leads to reorganization of the actin cytoskeleton and alterations in vascular permeability [9], which may also underlie the effect of Verge on ischemia-induced edema formation.

Intriguingly the present study found the stroke outcomes 24 or 72 hours after stroke were not significantly different between Verge KO and WT mice. The lack of neuroprotective effect of Verge deletion suggests that despite its rapid up-regulation in the ischemic hemisphere [9], and its possible contribution to edema formation, it does not appear to be critical to histological or behavioral outcomes in this model. Previous studies [14] have shown that edema formation after stroke does not necessarily correlate with infarct size. There are many possible explanations that may have contributed to the negative results on infarct size. Firstly Verge KO mice lack the Verge gene throughout development. As Verge is highly expressed during embryogenesis, compensatory pathways may have been activated to allow for the animal to survive without an overt phenotype. A conditional gene knockout may elucidate the effects of
Verge signaling in the adult brain [16]. Secondly, we found that Verge appears to be selectively expressed in the cerebral vasculature in WT mice, as seen by the lack of complete co-localization of Verge with CD31. It is possible that loss of Verge affected only a select vascular bed, which may contribute to the permeability changes. The lack of Verge expression in select vessels in the brain is provocative. Future studies will examine which vessels express Verge, at what time points after injury, and will attempt to identify the upstream regulators of Verge expression. This may be especially pertinent in clinical populations, as an increasing number of patients are receiving thrombolytics [17], with rates over $20 \%$ in some centers. Reducing endothelial permeability via Verge inhibition could potentially decrease the risk of hemorrhagic transformation after reperfusion. Examining these possibilities will be the subject of future studies. Thirdly, we did not evaluate chronic infarct size (i.e., weeks after stroke) or functional recovery after injury. It has been well documented that Verge is highly expressed during developmental angiogenesis [9], and it is possible that Verge signaling is important in triggering post- 


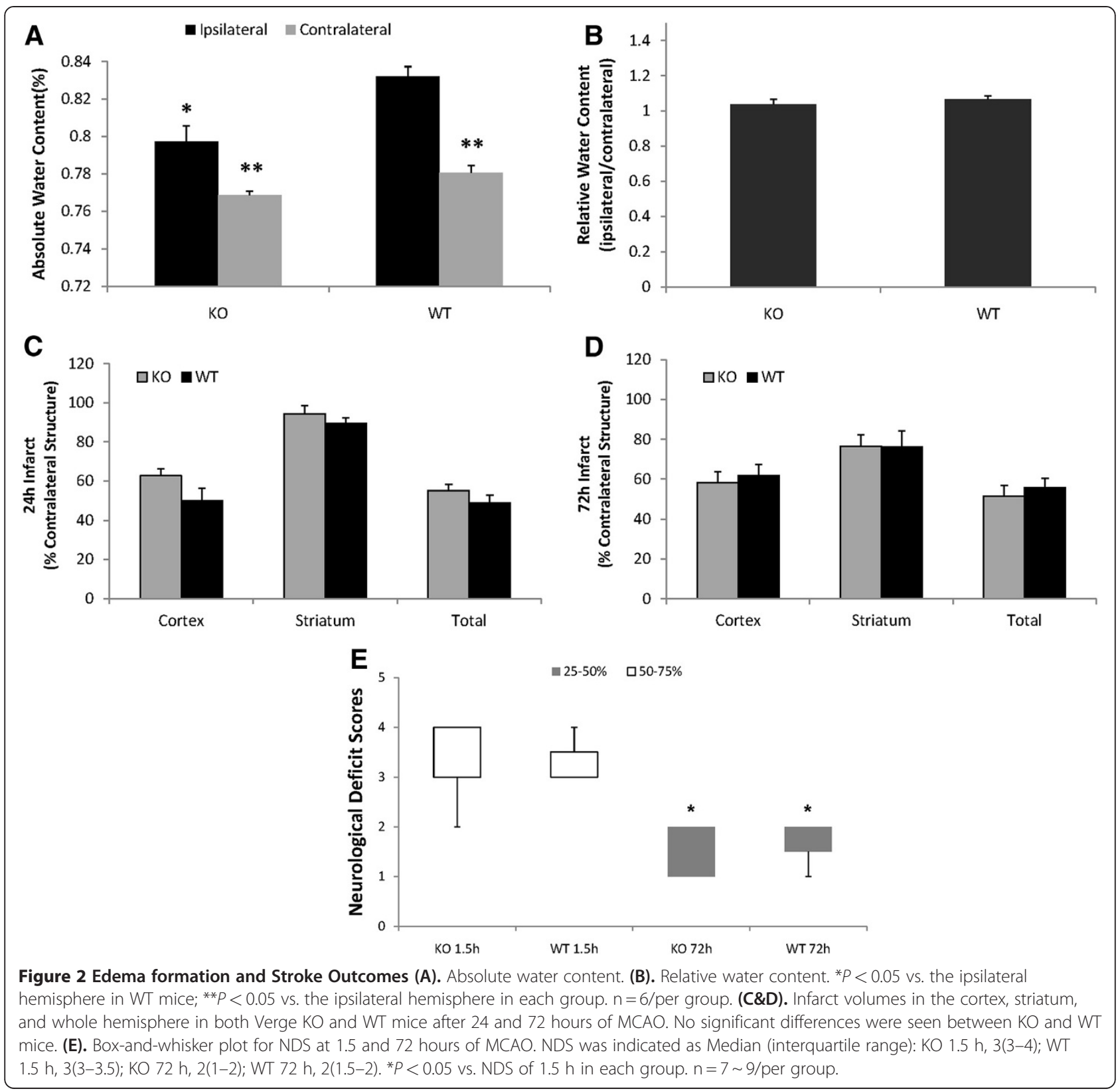

stroke angiogenesis and could contribute to behavioral recovery after injury. Post-stroke angiogenesis occurs at 3-4 days after stroke and continues for more than 21 days [18]. Therefore, further studies examining the effects of Verge in chronic ischemia models are necessary to confirm the lack of an effect of the loss of Verge on ischemic injury. Fourthly, although we evaluated large numbers of animals to confirm this effect and to avoid type II error and power analysis confirmed that no differences would be seen even with several hundred animals, cautious interpretation is always warranted in assessing negative studies. Despite these limitations, these studies suggest that loss of Verge does not have a significant effect on acute infarct size or behavioral deficits in this model at the time points examined.

\section{Competing interests}

The authors declare that they have no competing interests.

\section{Acknowledgements}

This work was supported by the NINDS (LDM NS050505 and NS055215) and an American Heart Association Scientist Development Grant (to FL ; 12SDG9030000).

\section{Author details}

'Department of Neuroscience, University of Connecticut Health Center, Farmington, CT 06030, USA. ²Department of Neuroscience, Johns Hopkins University, Baltimore, Maryland 21205, USA. ${ }^{3}$ Department Neurology, Johns Hopkins University, Baltimore, Maryland 21205, USA. ${ }^{4}$ Department Neurology, 


\section{Authors' contributions}

$\mathrm{FL}$ - Participation in the study design, conduction of experiments, statistical analysis, drafting of the manuscript, final edits of the manuscript. LCT Participation in the study design, conduction of experiments, statistical analysis, partially drafting of the manuscript. $J \mathrm{~L}$ - Conduction of experiments. $J R$ - Participation in the study design and coordination. PW - Participation in the study design and coordination. NZ - Conduction of experiments. LDM Study design, critically revising the manuscript, final edits to the manuscript.

All authors read and approved the final manuscript.

Received: 1 March 2012 Accepted: 8 June 2012

Published: 8 June 2012

\section{References}

1. Jung JE, Kim GS, Chen H, Maier CM, Narasimhan P, Song YS, Niizuma K, Katsu M, Okami N, Yoshioka H, et al: Reperfusion and neurovascular dysfunction in stroke: from basic mechanisms to potential strategies for neuroprotection. Mol Neurobiol 2010, 41(2-3):172-179.

2. Banks WA: Mouse models of neurological disorders: a view from the blood-brain barrier. Biochim Biophys Acta 2009, 1802(10):881-888.

3. Kahle KT, Simard JM, Staley KJ, Nahed BV, Jones PS, Sun D: Molecular mechanisms of ischemic cerebral edema: role of electroneutral ion transport. Physiology (Bethesda) 2009, 24:257-265.

4. Fowler T, Sen R, Roy AL: Regulation of primary response genes. Mol Cell 2011, 44(3):348-360.

5. O'Donnell A, Odrowaz Z, Sharrocks AD: Immediate-early gene activation by the MAPK pathways: what do and don't we know? Biochem Soc Trans 2012, 40(1):58-66.

6. Weinstein PR, Hong S, Sharp FR: Molecular identification of the ischemic penumbra. Stroke 2004, 35(11 Suppl 1):2666-2670.

7. Liu PK: Ischemia-reperfusion-related repair deficit after oxidative stress: implications of faulty transcripts in neuronal sensitivity after brain injury. J Biomed Sci 2003, 10(1):4-13.

8. Sandoval KE, Witt KA: Blood-brain barrier tight junction permeability and ischemic stroke. Neurobiol Dis 2008, 32(2):200-219.

9. Regard JB, Scheek S, Borbiev T, Lanahan AA, Schneider A, Demetriades AM Hiemisch $H$, Barnes CA, Verin AD, Worley PF: Verge: a novel vascular early response gene. J Neurosci 2004, 24(16):4092-4103.

10. Maallem S, Wierinckx A, Lachuer J, Kwon MH, Tappaz ML: Gene expression profiling in brain following acute systemic hypertonicity: novel genes possibly involved in osmoadaptation. J Neurochem 2008, 105(4):1198-1211.

11. Liu F, Schafer DP, McCullough LD: TTC, fluoro-Jade B and NeuN staining confirm evolving phases of infarction induced by middle cerebral artery occlusion. J Neurosci Methods 2009, 179(1):1-8.

12. Liu F, Akella P, Benashski SE, Xu Y, McCullough LD: Expression of Na-K-Cl cotransporter and edema formation are age dependent after ischemic stroke. Exp Neurol 2010, 224(2):356-361.

13. Liu F, Benashski SE, Persky R, Xu Y, Li J, McCullough LD: Age-related changes in AMP-activated protein kinase after stroke. Age (Dordr) 2012, 34(1):157-68.

14. Liu F, Yuan R, Benashski SE, McCullough LD: Changes in experimental stroke outcome across the life span. J Cereb Blood Flow Metab 2009, 29(4):792-802.

15. Liu F, Li Z, Li J, Siegel C, Yuan R, McCullough LD: Sex differences in caspase activation after stroke. Stroke 2009, 40(5):1842-1848.

16. Gerlai R: Gene targeting: technical confounds and potential solutions in behavioral brain research. Behav Brain Res 2001, 125(1-2):13-21.

17. Madej-Fermo OP, Staff I, Fortunato G, Abbott L, McCullough LD: Impact of emergency department transitions of care on thrombolytic use in acute ischemic stroke. Stroke 2012, 43(4):1067-1074.

18. Hayashi T, Abe K, Suzuki H, Itoyama Y: Rapid induction of vascular endothelial growth factor gene expression after transient middle cerebral artery occlusion in rats. Stroke 1997, 28(10):2039-2044.

doi:10.1186/2040-7378-4-12

Cite this article as: Liu et al.: Loss of vascular early response gene reduces edema formation after experimental stroke. Experimental \& Translational Stroke Medicine 2012 4:12.

\section{Submit your next manuscript to BioMed Central and take full advantage of:}

- Convenient online submission

- Thorough peer review

- No space constraints or color figure charges

- Immediate publication on acceptance

- Inclusion in PubMed, CAS, Scopus and Google Scholar

- Research which is freely available for redistribution 